\title{
Miniatured Planar Bandpass Filter Using Coupled Metamaterial Resonators
}

\author{
Gi-Rae Kim, Member, KIMICS
}

\begin{abstract}
In this article, new microstrip slow-wave bandpass filters using open loop resonator loaded with interdigital capacitive fingers is proposed. The filter features not only compact in size, but also exhibits spurious stop-band rejection. Filters of this type with elliptic function and Chebyshev response are demonstrated. There is good agreement between experimental and full-wave electromagnetic (EM) simulation results.
\end{abstract}

Index Terms - meandered microstrip; band-reject filter; the inter-digital loop resonator (IDCLLR); split ring resonator (SRR)

\section{INTRODUCTION}

IN many applications, miniaturized filters are highly sought after. Planar filters are preferred as they can be fabricated using low-cost printed circuit technology. A conventional microstrip filter with parallel-coupled halfwavelength resonators is too large to be used in the mobile, personal communication systems. Thus, it is desirable to develop a new microstrip bandpass filter structure that is compact and planar. Slow-wave structures have been used to reduce the size of the filter. Conventionally, a half-wave length open-loop resonator is used as a filter [1]. Microstrip line with both ends capacitively loaded with a pair of rectangular open-stubs [2] and a pair of triangular stub [3] has demonstrated to reduce the circuit size. On the other hand, capacitively loaded microstrip and CPW loop resonator has been investigated in $[4,5]$. Both resonators removed the internal part of the central strip of a conventional microstrip half-wavelength resonator. This effectively turns the standard resonator into a loop resonator. The fundamental resonance frequency might be estimated by assuming that its mean circumference equals the guided wavelength provided that the loop strip width is much smaller than the width of the associated half-wavelength resonator. The effect of this process on the slow wave is only small. To reduce the frequency of the resonator, it is loaded with capacitive fingers. The velocity reduction on this type of transmission line is control by the number of

\footnotetext{
Manuscript received March 16, 2011; revised March 28, 2011; accepted April 10, 2011.

Gi-Rae Kim is with the Department of Electronics Engineering, Silla University, Busan, 617-736, Korea, (Email: grkim@silla.ac.kr)
}

fingers within the narrow loop strip width. The resonator is a closed-loop structure. They can only be implemented in few filtering configurations. Conventionally, parallelcoupled line or end-coupled line filters design approaches can be used in this case [6]. In order to enhance filter performance, various filter configurations including elliptic or quasi-elliptic response would be desired.

In this paper, we proposed to use the inter-digital capacitive fingers, which loaded in the open-loop resonator to achieve cross-coupled slow-wave microstrip elliptic function and Chebyshev filters. In [4, 5], the resonator is a transmission line modified with etched fingers to achieved slow-wave effect and miniaturization. Here, the attempt is to provide close capacitive coupling between two arms of the transmission line using added fingers to create a new miniaturize resonator. This method of enhancing the capacitively loaded microstrip open-loop resonator enables the presented

\section{METAMATERIAL RESONATOR}

The quasi-static resonant angular frequency $\omega_{0}=1 / \sqrt{L_{0} C_{0}}$ of IDSRR depends on the total inductance $\mathrm{L}_{0}$ of the IDSRR approximated by that of a single close ring; and the quasi-static equivalent capacitance $\mathrm{C}_{0}$ of the interdigital capacitor [10]

$$
C_{e p}(p F)=\frac{\varepsilon_{r} 10^{-3} K(k)}{18 \pi K^{\prime}(k)}(N-1) l
$$

where $\varepsilon_{\mathrm{r}}$ is the effective permittivity of microstrip line with the width $\mathrm{d}$, the number of fingers $\mathrm{N}$, and the finger length $1 ; \mathrm{K}(\mathrm{k})$ and $\mathrm{K}^{\prime}(\mathrm{k})$ is complete elliptic function of the first kind and its complementary function with modulus $\mathrm{k}=\tan ^{2}[\mathrm{~d} \pi / 4(\mathrm{~d}+\mathrm{s})]$. Obviously, the equivalent capacitance of IDSRR depends on so many parameters, resulting in more freedom in design. Fig. 2 shows the structure of IDSRR-based and SRR-based resonator. A meandered microstrip line of $50 \mathrm{ohm}$ and the embedded IDSRR/SRR are etched on the same surface of substrate (relative permittivity $\varepsilon_{\mathrm{r}}=2.17$, thickness $\mathrm{h}=0.7874 \mathrm{~mm}$ ). The width of microstrip line is $\mathrm{w}=2.4 \mathrm{~mm}$ corresponding to $50 \mathrm{ohm}$ of characteristic impedance. The spacing between IDSRR/SRR and line is $\mathrm{r}=0.2 \mathrm{~mm}$. The size of IDSRRR/SRR is $\mathrm{a}=10.45 \mathrm{~mm}, \mathrm{~b}=11.95 \mathrm{~mm}, \mathrm{c}=1.2 \mathrm{~mm}$, $\mathrm{g}=\mathrm{e}=0.2 \mathrm{~mm}, \mathrm{~d}=\mathrm{s}=0.3 \mathrm{~mm}, \mathrm{l}=7.6 \mathrm{~mm}, \mathrm{f}=\mathrm{h}=\mathrm{k}=0.6 \mathrm{~mm}$, and number of fingers $\mathrm{N}=1$ [refer Fig. 1(a) and 1(b)]. 

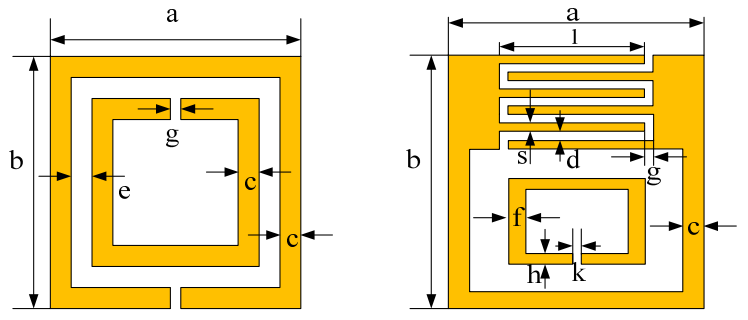

Fig. 1. (a) the conventional square open-loop resonator (b)IDSRR.
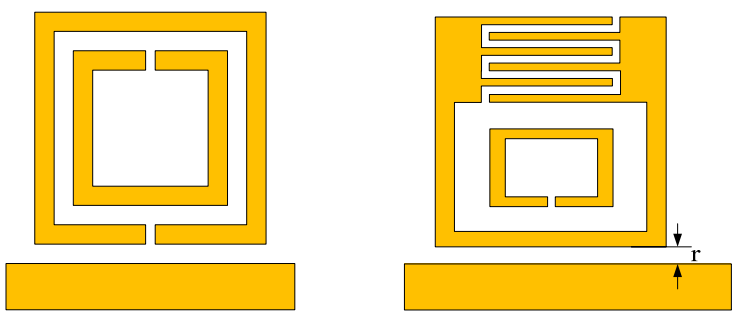

Fig. 2. Structure of single-stage resonator (a) SRR-based structure (b) IDSRR-based structure.

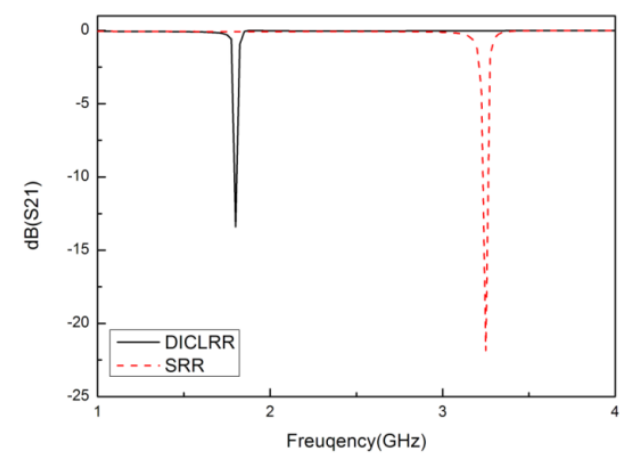

Fig. 3. Insertion loss frequency responses of single-stage resonator.

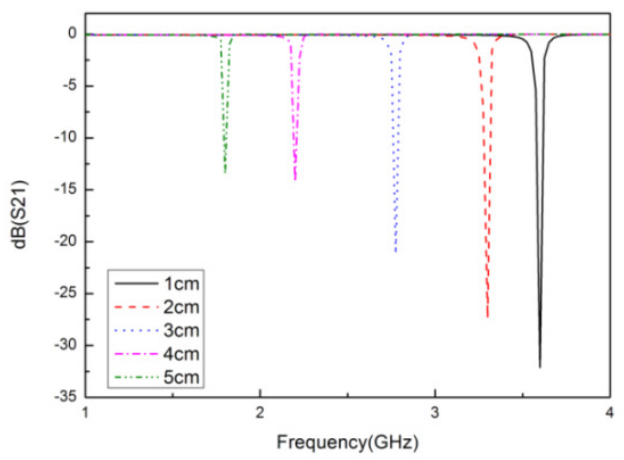

Fig. 4. Simulated insertion-loss responses of IDCLLRbased filter varied with inter-digital length.
The simulated (using HFSS V10 software) frequency responses for both structures are described in Fig. 3. Where [1] the resonant frequency of IDSRR-based meandered filter $(\sim 1.8 \mathrm{GHz})$ is lower than that of SRRbased one $(\sim 3.3 \mathrm{GHz})$, i.e., the resonator size can be reduced by compact-ratio $0.54: 1$ for the same operated frequency [2]. Obviously, the latter has higher rejection level and wider stop-bandwidth than the former. This is because the SRR is the structure of double rings, so that larger induced current can be excited at resonance, as a result, higher rejection level can be obtained in SRRbased filter [3]. In comparison, a SRR-based singlestage (a pair of SRRs) but non-meandered filter performs $\sim-10 \mathrm{~dB}$ maximal insertion-loss at resonant frequency [6], it clearly displays the improvement due to stronger coupling in meandered structure [4]; some systematic errors ( $\sim 6 \%$ for resonant frequency) exist between simulation and measurement. Moreover, if changed the inter-digital length 1 but keep fixed sizes s, $\mathrm{d}$, and $\mathrm{g}$ [refer Fig. 1(b)], it is easier to design an IDCLLR with desired frequency.

\section{FILTER CHARACTERISTICS}

The filter having only one pair of transmission zeros (or attenuation poles) at finite frequencies gives much improved skirt selectivity, making it a viable intermediate between the Chebyshev and elliptic-function filters, yet with little practical difficulty of physical realization. The transfer function of this type of filter is

$$
\begin{gathered}
\left|S_{21}(\Omega)\right|^{2}=\frac{1}{1+\varepsilon^{2} F_{n}^{2}(\Omega)} \\
\varepsilon_{r}=\frac{1}{\sqrt{10^{-\frac{L_{R}}{10}}-1}} \\
F_{n}(\Omega)=\cosh \left\{(n-2) \cosh ^{-1}(\Omega)+\cosh ^{-1}\left(\frac{\Omega_{a} \Omega-1}{\Omega_{a}-\Omega}\right)+\cosh ^{-1}\left(\frac{\Omega_{a} \Omega+1}{\Omega_{a}+\Omega}\right)\right\}
\end{gathered}
$$

where $\Omega$ is the frequency variable that is normalized to the passband cut-off frequency of the lowpass prototype filter, $\varepsilon_{\mathrm{r}}$ is a ripple constant related to a given return loss $\mathrm{L}_{\mathrm{R}}=20 \log |\mathrm{S} 11|$ in $\mathrm{dB}$, and $\mathrm{n}$ is the degree of the filter. It is obvious that $\Omega= \pm \Omega_{\mathrm{a}}\left(\Omega_{\mathrm{a}}>1\right)$ are the frequency locations of a pair of attenuation poles. Note that if $\Omega_{\mathrm{a}} \rightarrow \infty$ the filtering function $F_{n}(\Omega)$ degenerates to the familiar Chebyshev function. The transmission frequency response of the bandpass filter may be determined using frequency mapping, i.e.,

$$
\Omega=\frac{1}{F B W} \cdot\left(\frac{\omega}{\omega_{0}}-\frac{\omega_{0}}{\omega}\right)
$$

in which $\omega$ is the frequency variable of bandpass filter, $\omega_{0}$ 
is the mid-band frequency and FBW is the fractional bandwidth. The locations of two finite frequency attenuation poles of the bandpass filter are given by

$$
\begin{gathered}
\omega_{a 1}=\omega_{0} \frac{-\Omega_{a} F B W+\sqrt{\left(\Omega_{a} F B W\right)^{2}+4}}{2} \\
\omega_{a 2}=\omega_{0} \frac{\Omega_{a} F B W+\sqrt{\left(\Omega_{a} F B W\right)^{2}+4}}{2}
\end{gathered}
$$

\section{FILTER DESIGN THEORY}

The design parameters of bandpass filters, i.e., the coupling coefficients and external quality factor in Fig. 5, can be determined in terms of circuit elements of a lowpass prototype filter of Fig. 6, which consists of lumped capacitors and ideal admit The design parameters of bandpass filters, i.e., the coupling coefficients and external quality factor in Fig. 5, can be determined in terms of circuit elements of a low-pass prototype filter of Fig. 6, which consists of lumped capacitors and ideal admittance inverters. The relationships between the bandpass design parameters and the low-pass elements are

$$
\begin{gathered}
Q_{e i}=Q_{e o}=\frac{C_{1}}{F B W} \\
M_{n, n-1}=M_{N-n, N-n+1} \\
=\frac{F B W}{\sqrt{C_{n} C_{n+1}}}, \text { for } \mathrm{n}=1 \text { to } \frac{N}{2} \\
M_{m, m+1}=\frac{F B W \cdot J_{m}}{C_{m}}, \text { for } \mathrm{m}=\frac{N}{2} \\
M_{m-1, m+2}=\frac{F B W \cdot J_{m-1}}{C_{m-1}}, \text { for } \mathrm{m}=\frac{N}{2}
\end{gathered}
$$

where denotes the fractional bandwidth of the bandpass filter, is the capacitance of the lumped capacitor and is the characteristic admittance of the inverter, and is the degree of the filter. To find the element values of a low-pass prototype, one may use an approximate synthesis method described in [2]. This method is simple, but it suffers from inaccuracy and can even fail for highly selective filters that require moving the attenuation poles closer to the cutoff frequencies of the passband. However, the exact synthesis process is rather complicated and time consuming because there does not exist any closed-form formulas for the element values. The values of the attenuation pole frequency were chosen such that they cover a wide range of practical designs for selective microstrip bandpass filter responses. Referring to Fig. 1, the side lobe at the stopband would be too high if is smaller than the given values.

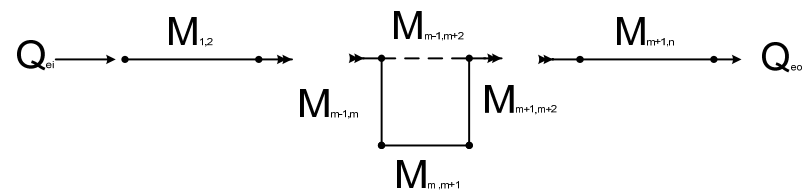

Fig. 5. General coupling structure of the bandpass filter with a single pair of finite-frequency zeros.

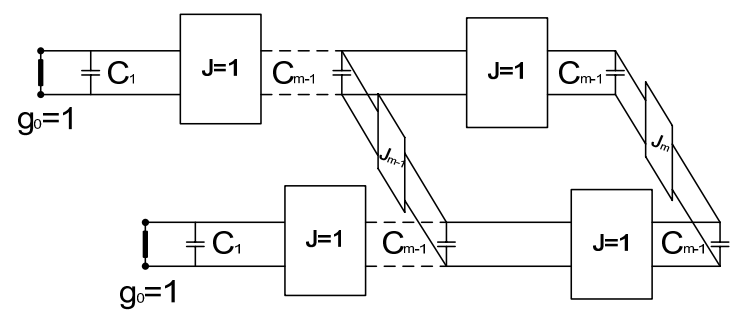

Fig. 6. Lowpass prototype filter for the filter synthesis.

In this paper, we choose second-order structure with two transmission zeros. The first building block is a second-order structure in which the source is directly coupled to the load in order to generate the two transmission zeros. Here, the dark disks represent the resonator in the low-pass prototype.

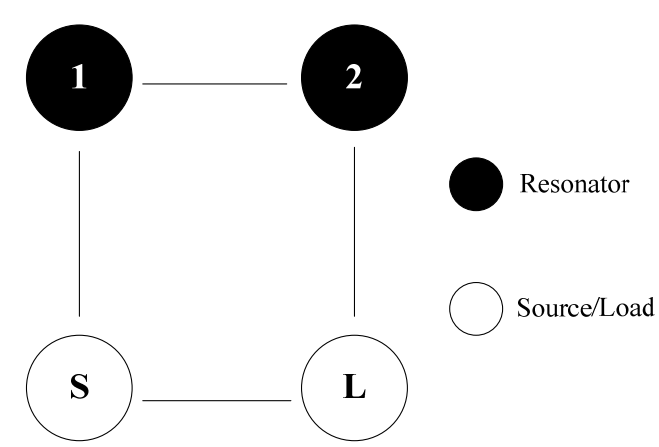

Fig. 7. Coupling and routing scheme of second-order building blocks.

\section{COUPLING COEFFICIENTS}

The new square open-loop resonator loaded with interdigital fingers depicted in Fig. 1(b) is used to demonstrate a cross coupled elliptic function and a Chebyshev response filter. Both the 4-pole filters with center frequency at $1.8 \mathrm{GHz}$ and a fractional bandwidth bandwidth of $100 \mathrm{MHz}$ were designed and fabricated on Teflon substrate thickness of $0.7874 \mathrm{~mm}$ with relative dielectric constant $\varepsilon_{\mathrm{r}}$ of 2.12. EM simulator HFSS V10 
was used to simulate and design the filter. Fig. 3 shows the characterized three basic coupling structures referred to as the magnetic coupling, electric coupling and mixed coupling. The coupling coefficient $\mathrm{K}_{\mathrm{ij}}$ of any pair of adjacent resonators can be determined by Eq. (12):

$$
K_{i j}= \pm \frac{f_{p 2}^{2}-f_{p 1}^{2}}{f_{p 2}^{2}+f_{p 1}^{2}}
$$

where $f_{p 1}$ and $f_{p 2}$ are the lower and higher split resonant frequencies of a pair of coupled resonators when they are decoupled from the rest. The 4-pole cross coupled filter could be designed using the method described in [8], from which the lump-element values were determined as $\mathrm{K}_{12}$ $=0.0322, \mathrm{~K}_{23}=0.0393, \mathrm{~K}_{34}=0.03173$, and $\mathrm{K}_{41}=0.030$.

Fig. 8 and 9 show the layout, simulated and measured performances of filter. The spacing designated in the cross- coupled filter is as follows: $\mathrm{a}_{12}=1 \mathrm{~mm}, \mathrm{a}_{23}=2.0$ $\mathrm{mm}, \mathrm{a}_{34}=1 \mathrm{~mm}$ and, $\mathrm{a}_{14}=1.3 \mathrm{~mm}$. The effects on the center frequency and bandwidth are negligible as long as the gap tuning is small. The measured passband insertion loss is $-3 \mathrm{~dB}$, good agreement with the simulation. Fig. 9 shows the simulated and measured characteristics of the filters. Both filters exhibit a good rejection at second harmonic as predicted. Fig. 10 shows the prototype of bandpass filter.

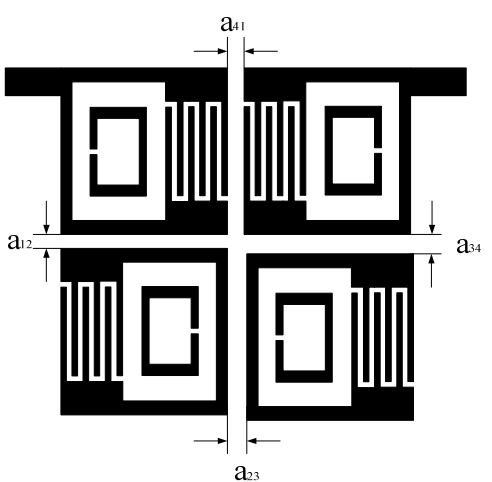

Fig. 8. Four-pole IDCLLR-based meandered bandpass filter.

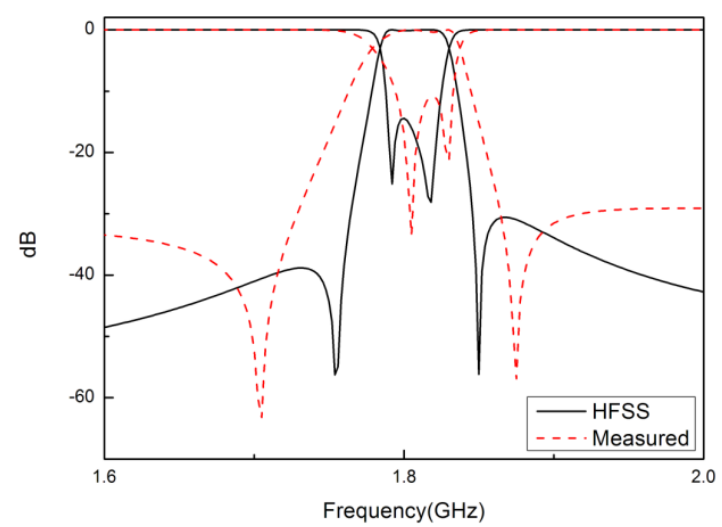

Fig. 9. Simulated and measured frequency response of insertion/return-loss.

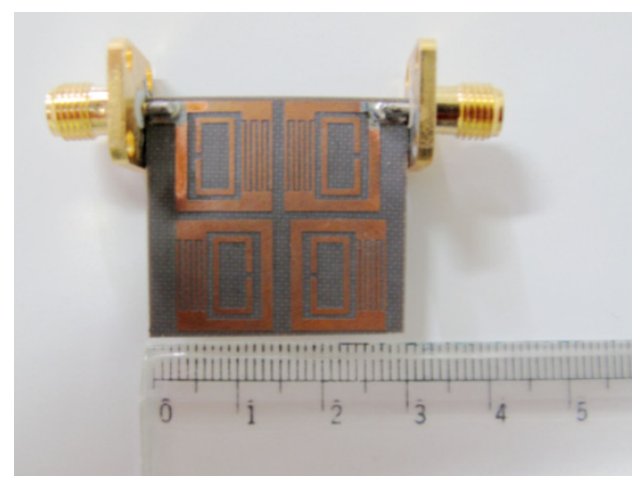

Fig. 10. Fabricated prototype

\section{CONCLUSION}

New miniaturized slow-wave open-loop resonator structure loaded with inter-digital capacitive fingers is proposed. We have demonstrated a 4-pole cross-coupled elliptic function filter and a Chebyshev filter of this type. Both filters achieved compact miniaturization and stopband spurious rejection. Simulated and measured results are in good agreement.

\section{REFERENCES}

[1] J.S. Hong and M.J. Lancaster, "Couplings of microstrip square open-loop resonators for cross-coupled planar microwave filters", IEEE Trans. Microwave Theory Tech., vol. 44 , pp. 2099-2109, 1996.

[2] J.S. Hong and M.J. Lancaster, "Theory and experiment of novel microstrip slow-wave open-loop resonators filters", IEEE Trans. Microwave Theory Tech., vol. 45, pp. 2358-2365, 1997.

[3] J.J. Yu, S.T. Chew, M.S. Leong, and B.L. Ooi, "New class of microstrip miniaturized filter using triangular stub", Elec. Lett., vol 37, pp. 1169-1170, 2001.

[4] J.S. Hong and M.J. Lancaster, "Capacitively loaded microstrip loop resonator", Elec. Lett., vol. 30, pp. 1494-1495, 1994.

[5] A. Gorur and C. Karpuz, "Experimental study on characteristics of loaded CPW resonators", Microwave Opt. Technol. Lett., vol. 21 pp, 199-201, 1999.

[6] J.S. Hong and M.J. Lancaster, "Edge coupled microstrip loop resonators with capacitive loading”, IEEE Microwave Guided Wave Lett., vol. 5, pp. 87-89, 1995.

[7] Sonnet Software Inc., Sonnet simulator.

[8] J.S. Hong and M.J. Lancaster, "Design of highly selective microstrip bandpass filters with a single pair of attenuation poles at finite frequencies” , IEEE Trans. Microwave Theory Tech., vol. 48, pp. 1098-1107, 2000.

[9] J.S. Hong, "Microstrip tapped line filter design", IEEE Trans Microwave Theory Tech., vol. 27 , pp. 44-45, 1979.

Girae Kim received the B.S., M.S. in Electronic Engineering from the Sogang University, Seoul, Korea, in 1986, 1988, respectively. He also received Ph.D. from Kyungnam University, Korea in 1999. From 1988 to 1993 he was a researcher in Communication Research Center of Samsung Electronics Co. Ltd. Since 1999, he has been on the faculty of Electronics Engineering Department at the Silla University, Busan, Korea. 